\title{
Vibration Suppression and Precision Positioning of a Piezoelectric Beam with Graded Properties
}

\author{
Eman Eshraqi ${ }^{a *}$, Morteza Shahravi ${ }^{b}$ and Milad Azimi ${ }^{c}$ \\ ${ }^{a}$ Researcher. Tehran, Iran \\ ${ }^{b}$ Malek Ashtar University of Technology, Tehran, Iran \\ ${ }^{c}$ Space Research Institute, Tehran, Iran \\ *E-mail address: eman.eshraqi@chmail.ir
}

\begin{abstract}
This paper focuses on the development of an active control system for simultaneous precision positioning and vibration suppression of a functionally graded piezoelectric material (FGPM) beam, using finite element analysis (FEA). The developed environment includes three modules: structural modelling, controller design, and dynamic analysis of the smart structure. The gain selection for the controller is based on achieving the best positioning accuracy and vibration suppression simultaneously. It is demonstrated that the FEA model with integrated controller is able to reach the desired position in a relatively small time.
\end{abstract}

Keywords: FGPM; Vibration Suppression; Precision Positioning; Finite Element Analysis.

\section{Introduction}

Piezoelectric materials have been widely used as actuators and sensors in smart structures and systems for their effective capability of conversion between mechanical and electric fields. Piezoelectric actuators are often made by bonding piezoelectric sheets to get better performance; however the bonding will cause severe interfacial stress concentration, and as a result triggers the initiation and propagation of micro-cracks near the interface, and early failure of the devices [1]. Functionally graded materials (FGMs) have no distinct interfaces and the material properties vary in in one (or more) direction(s), which may enhance their mechanical characteristics and make them suitable for various applications in harsh environments [2]. A class of piezoelectric materials with graded properties, called functionally graded piezoelectric materials (FGPMs), has been developed to remove issues of the traditional layered piezoelectric elements and thermo-electro-mechanical properties of the structures made from FGPMs have been extensively analysed by many researchers [3-5].

Smart structures with simultaneous precision positioning and vibration suppression capabilities have many potential applications in military and space structures to enhance their structural performance. However, this is more challenging than precision positioning or vibration suppression alone, since the control design must take care of static and dynamic features of structures including positioning accuracy, rapidness of tracking, and vibration attenuation. Up to now, much attention has been paid to the design, analysis, experiments and industrialization of active vibration/noise control of intelligent structures with piezoelectric sensors and actuators or [6-9] or to the analysis and design of static and dynamic shape control and positioning [10-13]. This work focuses on simultaneous precision positioning and vibration suppression of smart structures made of FGPM .

This paper consists of finite element modelling of smart beam, PID control scheme and its implementation in the ABAQUS FE commercial code. Furthermore, parameter study of the 
controller for simultaneous precision positioning and vibration suppression of structure followed by the conclusion is discussed here.

\section{Finite Element Modelling and Controller Integration}

For this study, the system is selected as a cantilevered bimorph functionally graded beam with fixed boundary condition for one end. The method used to model the FGPM beam is based on assumption of the FGPM structure being a laminated beam which consists of a number of laminae. The material properties within each laminate are assumed to be invariant. The beam is modelled by two morphs of FGPM assuming each morph includes finite number of layers within each the material properties are constant. The properties vary according to the relations $s_{i j}=s_{i j}^{0} F(y), d_{i j}=d_{i j}^{0} F(y)$, and $\varepsilon_{i j}=\varepsilon_{i j}^{0} F(y)$ where $s_{i j}, d_{i j}$, and $\varepsilon_{i j}$ are the elastic, piezoelectric , and dielectric constansts respectively $; s_{i j}^{0}, d_{i j}^{0}$, and $\varepsilon_{i j}^{0}$ are the corresponding values at plane where $F(y)=1 . F(y)$ is the graded function given by $F(y)=\exp \left(\frac{\alpha y}{h}\right)$ where $\alpha$ is the graded index , $y$ is distance from bottom surface of the morph and $h$ is the thickness of each morph. It is worth mentioning that both morphs have the same material properties and the only difference is that they have opposite polarization directions (one in $+y$ direction and the other in $-y$ direction). The material properties at $y=0$ are those of PZT-4 given in [1] and shown in Table 1. A graded index of value 3 is used herein. Fig. 1 shows a schematic diagram of the model considered in this study.

Table 1: Material properties of PZT-4

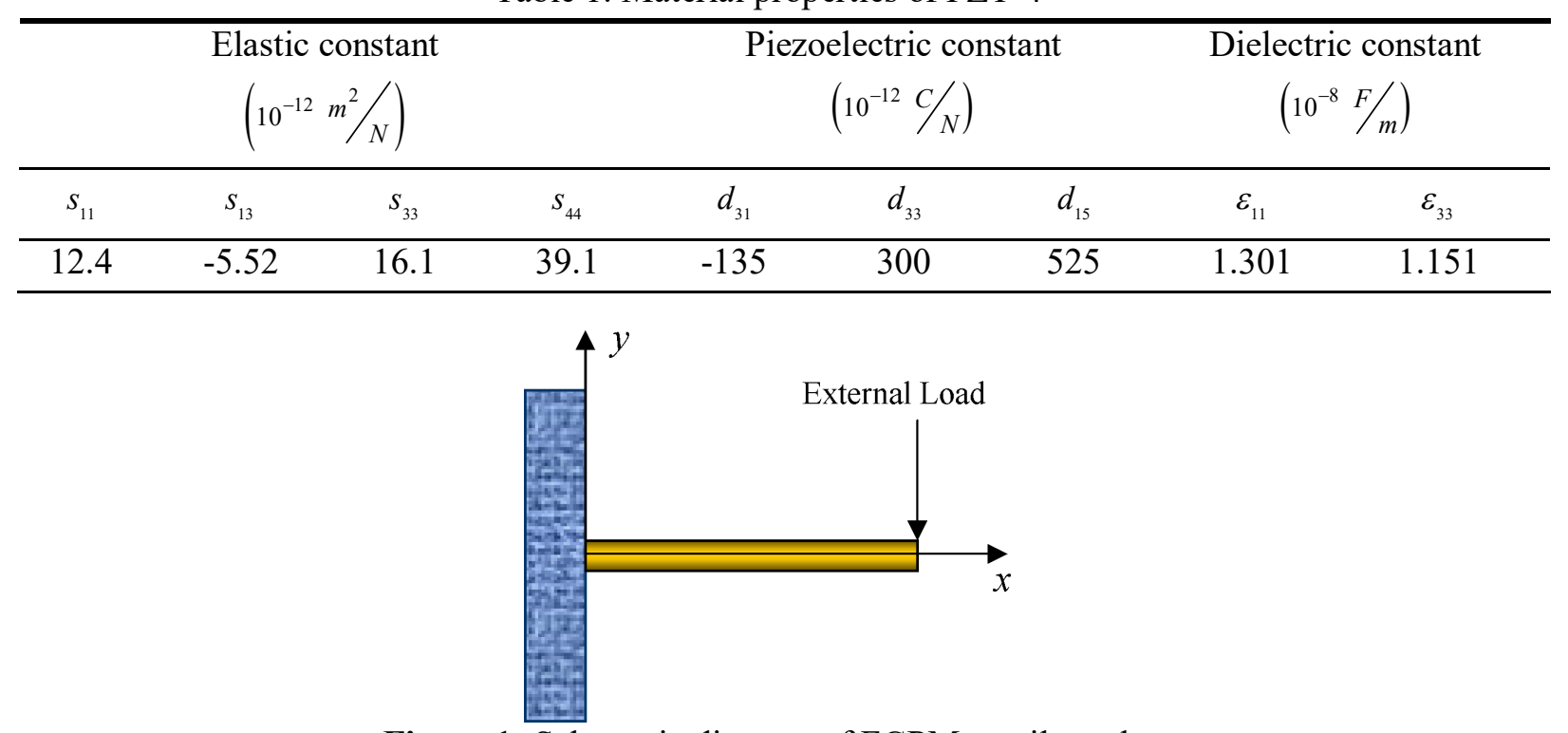

Figure 1: Schematic diagram of FGPM cantilever beam

This Active FGPM Beam is modeled using ABAQUS FE code. A PYTHON script is developed for such simulation which starts with the definition of the variables for the FGPM structure dimensions as $25 \mathrm{~cm}$ in length, $5 \mathrm{~cm}$ in width, and $1 \mathrm{~cm}$ in thickness. Next, the two dimensional mechanical properties and electromechanical properties are defined. CPE8RE element [14] is used for the FGPM beam meshing with variation of material properties implemented in the FE model using USDFLD subroutine. A FE modal analysis is also performed and the beam first natural frequency is evaluated to be $37.83 \mathrm{~Hz}$. The time step interval needed for the transient analysis is selected as 0.002 
seconds for this study. The structural damping used for the FGPM beam is assumed to be a constant value of 0.001 .

A closed-loop control block diagram for smart structure control is shown in Fig. 2. The controller type can be selected based on the performance requirement, complexity in design and implementation, etc. In this paper, PID controller is selected due to the fact that it is simple and does not require controlled plant information. In Fig. 2, $e$ represents the tracking error, which is defined as the difference between the desired output $(R)$ and the actual output $(Y)$, i.e. $e=R-Y$. In this work, actual output $(Y)$ is represented by the value of the displacement in the $y$-direction corresponding to the beam tip (see Fig. 1), and it is calculated at each time step during the transient analysis. The error signal is sent to the controller, where both the integral and the derivative of this error signal are computed. The required actuator voltage, i.e., the control signal represented by $V_{a}$ in Fig. 2, can be calculated by using Eq. (1), which includes proportional $(P)$, integral $(I)$, and derivative $(D)$ parts:

$$
V_{a}=K_{p} e+K_{i} \int e d t+K_{d} d e / d t
$$

where $K_{p}, K_{i}$, and $K_{d}$ are the proportional gain, integral gain, and derivative gain of a PID controller, respectively.

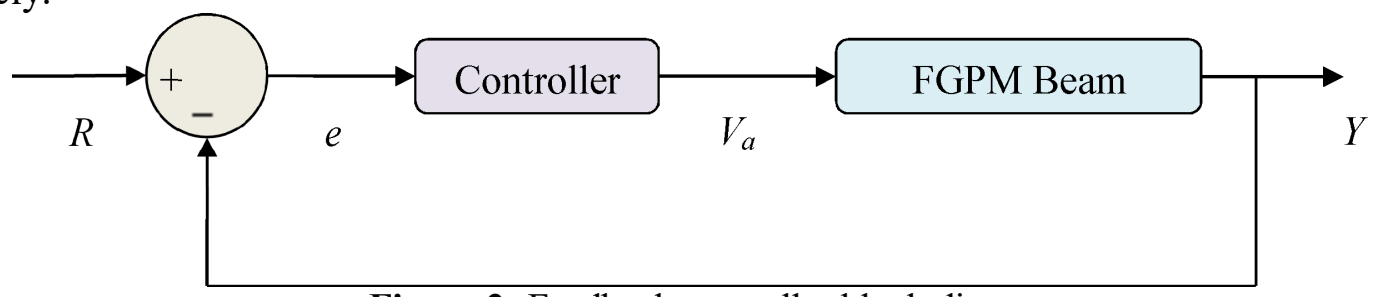

Figure 2: Feedback controller block diagram

\section{Selection of controller gains}

This section focuses on the design of a controller that provides the best simultaneous vibration suppression and precision positioning in various disturbances such as free vibration, sinusoidal disturbances at different frequencies, and random disturbance.

In general, the beam tip must be deflected to a desired position and the vibration at the beam tip should be suppressed simultaneously. The beam vibration is either the free vibration caused by the beam move from one place to another or the forced vibration caused by the applied external forces at the beam tip. Here the external loads are in forms of sinusoidal loads with different frequency values as well as a random force. The proper gain values of the controller will be determined based on the best positioning accuracy and vibration suppression capability. The beam tip and its corresponding displacement in the $y$-direction serve as the sensor location and sensor signal, respectively. To compare the vibration reduction capability of the controller, a certain DC voltage is applied to deflect the beam tip to a desired position (i.e., some specific displacement in the $y$-direction) and meanwhile to excite the free vibration of the beam. It should be noted that the $\mathrm{DC}$ voltage is different from the controller output, and is not necessary to know in practice. The introduction of the DC voltage is solely for comparison purpose. This DC voltage is determined by applying it to the beam to achieve the same displacement as the desired displacement $(R)$ at the beam tip. The steps for determining the DC voltage are the following: (a) supplying a dummy voltage, represented by variable $\left(V_{d}\right)$, to top surface of the beam and measuring the displacement in the $y$-direction, represented by variable $\left(D_{d}\right)$; (b) using linearity assumption and Eq. (2) to calculate the DC voltage $\left(V_{d p}\right)$ needed to deflect the 
beam tip to the desired displacement $(R)$. For example, the calculated DC voltage to deflect the beam tip by $0.1 \mathrm{~mm}$ is 375 volts.

$$
V_{d p}=\left(R / D_{d}\right) V_{d}
$$

After applying the DC voltage $V_{d p}$ to the beam, the beam tip will deflect to the desired displacement with some vibration and this displacement including static and dynamic parts is recorded for comparison purposes. The static part of the displacement is the desired position and the dynamic part is the free vibration of the structure or the free vibration plus the forced vibration if an external force is also applied at the beam tip. These records are shown in Figs. 3 and 4.

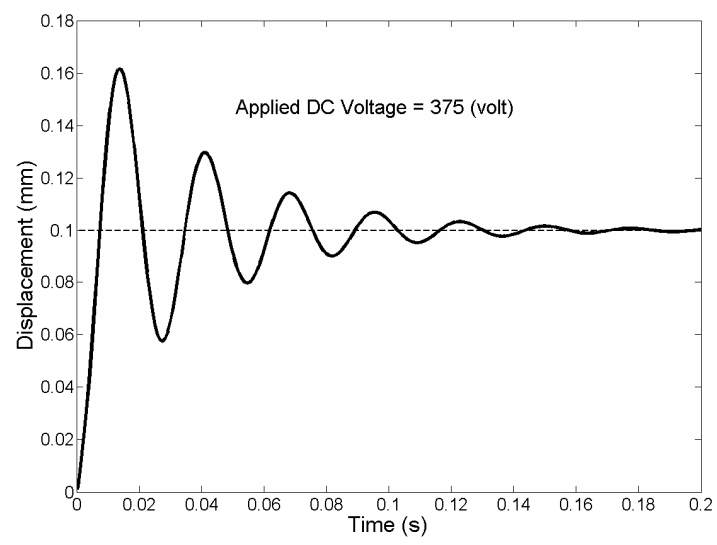

Figure 3: Free vibration of the beam

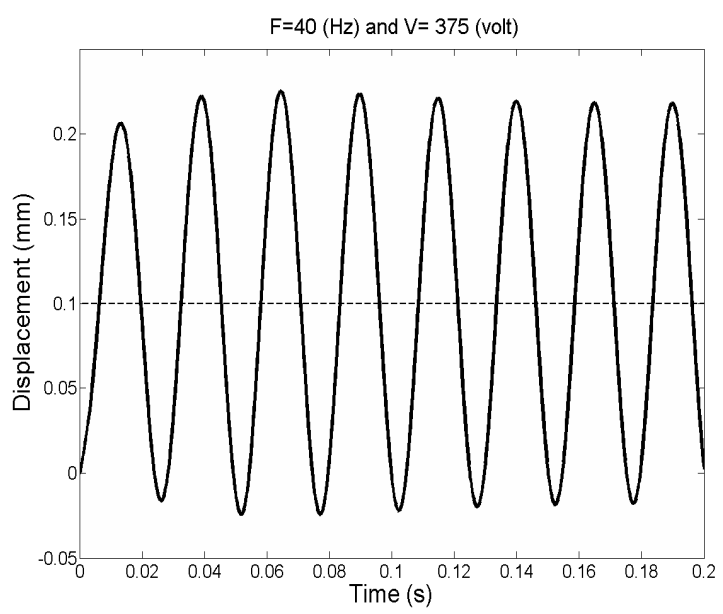

Figure 4: Forced vibration of the beam

\subsection{Characteristics of the proportional gain $\left(K_{p}\right)$}

To determine the proper gain values for the controller, the tip of the beam is expected to deflect 0.1 $\mathrm{mm}(R)$ in the $y$-direction. Fig. 3 shows the uncontrolled and free vibration transient response when the beam tip is deflected by $0.1 \mathrm{~mm}$. The effect of different proportional gain values on the vibration suppression of the beam tip is shown in Fig. 5. As mentioned earlier, the tracking error is the difference between the desired displacement $(R)$ and the actual output (i.e., the value of the displacement sensor at each time step). The results indicate that the $K_{p}$ has effects on both reducing the overshoot and the rising time. Also increasing the value of $K_{p}$ has effects on reducing, but never 
eliminating, the steady-state error. However, too large $K_{p}$ causes large free vibration oscillation which means that the stability margin of the system is getting smaller. $K_{p}$ value of $-4.5 \times 10^{5}$ is selected here.

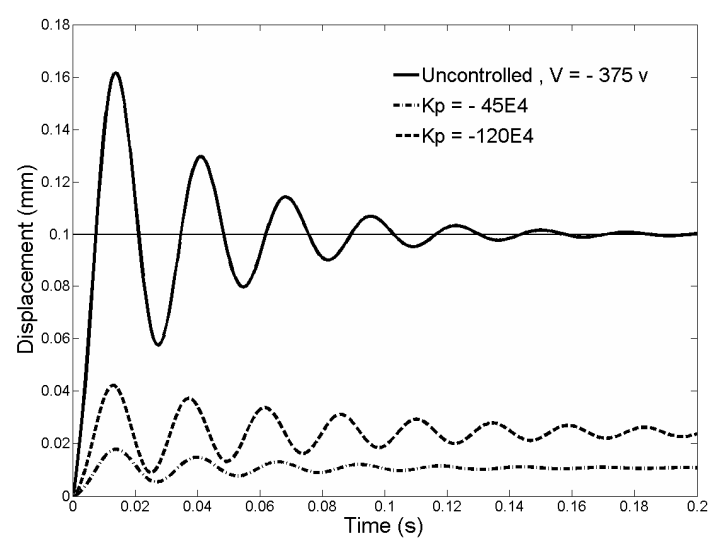

Figure 5: Effect of $K_{p}$

\subsection{Characteristics of the integral gain $\left(K_{i}\right)$}

To examine the characteristics of the integral gain $\left(K_{i}\right)$, different $K_{i}$ values are assigned as the selected $K_{p}$ value is kept constant. Fig. 6 demonstrates the behaviour of $K_{i}$ on achieving the desired position and performing vibration suppression, indicating $K_{i}$ has the effect of eliminating the steadystate error as well as decreasing the settling time. It should be noted that, incorrect $K_{i}$ value (for example very large values) makes the transient response worse or even unstable. The $K_{i}$ value of $1.2 \times 10^{8}$ is selected here.

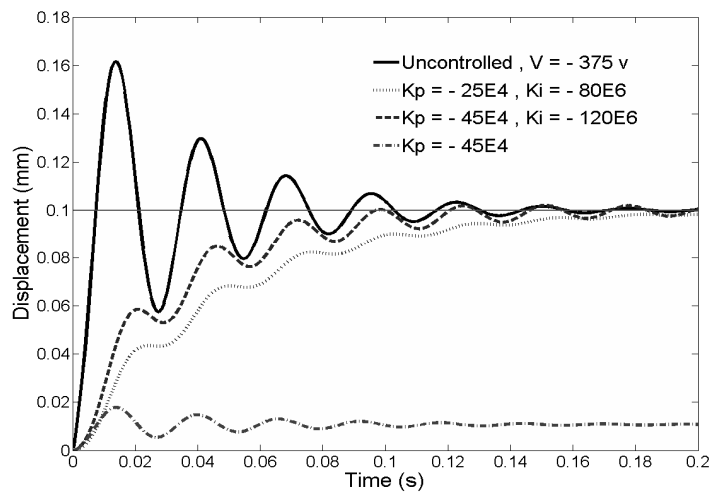

Figure 6: Effect of $K_{i}$

\subsection{Characteristics of the derivative gain $\left(K_{d}\right)$}

Finally, the effect of the derivative gain $\left(K_{d}\right)$ is studied while the selected gain values for the $K_{p}$ and $K_{i}$ are kept constant. Fig. 7 demonstrates the behaviour of different $K_{d}$ values. It is clear that the proper $K_{d}$ value has the effect of increasing the stability of the system in forms of improving the controlled transient response by decreasing the settling time, eliminating the steady-state error to zero, and reducing both the overshoot and the rising time, while Incorrect selection of $K_{d}$ value leads the system to become unstable. The $K_{d}$ value of $-4 \times 10^{4}$ is selected here. 
Hence, the selected best gains for the controller are as following: $K_{p}=-4.5 \times 10^{5}, K_{i}=-1.2 \times 10^{8}$, and $K_{d}=-4 \times 10^{4}$. The results demonstrate that the controller can reach the desired position while simultaneously performing vibration attenuation. The percentage of steady-state error is zero at settling time of 0.12 seconds as depicted in Fig. 7.

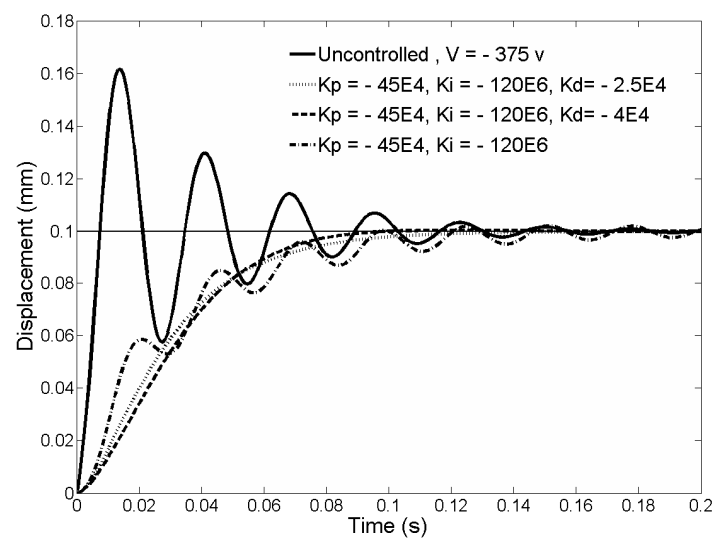

Figure 7: Effect of $K_{d}$

\subsection{Performance evaluation of the controller due to external sinusoidal and random forces}

The controller performance effectiveness is evaluated when the beam tip is deflected by a specific amount and simultaneously is subjected to different types of external loads such as sinusoidal with different frequencies as well as random force. Different desired positions $(R)$ with magnitudes of 0.1 or $0.2 \mathrm{~mm}$ are also employed in the formula measuring the tracking error. Effect of the controller on achieving a proper positioning and vibration suppression when the beam tip is subjected to a sinusoidal excitation force at $40 \mathrm{~Hz}$ and an imposed desired position of $0.2 \mathrm{~mm}$ is shown in Fig. 8a. A similar simulation is also performed when the desired position is set at $0.1 \mathrm{~mm}$. The results indicate that as the desired position value increases from 0.1 to $0.2 \mathrm{~mm}$ the corresponding control response has smaller oscillation meaning that the controller performs better vibration suppression initially and almost identically at the later time. For position control, the tracking error $(e)$ would be the difference between the desired displacement $(R)$ and the actual displacement output $(Y)$. It should be noted that the actual output is the beam tip displacement calculated for each time step during the transient analysis.

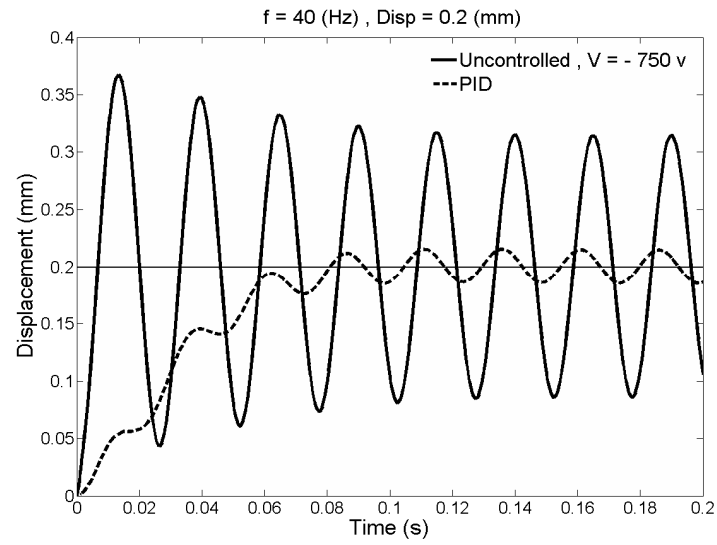

(a)

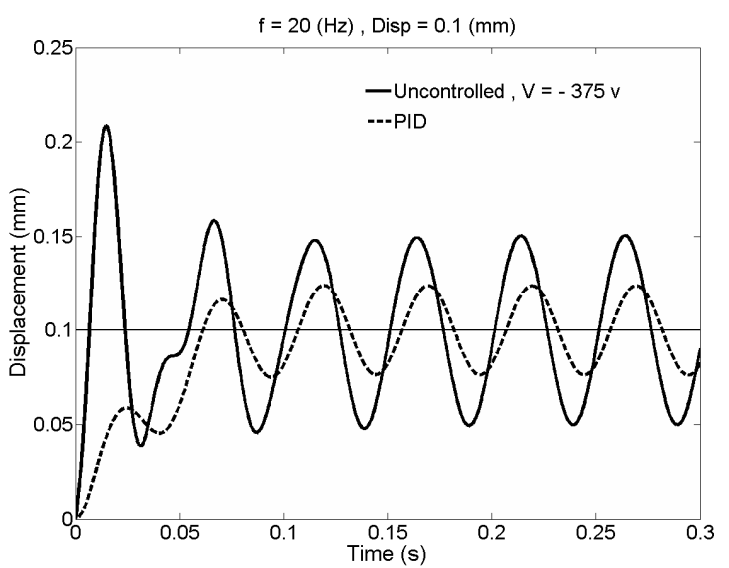

(b)

Figure 8: Effects of the controller on vibration suppression of the beam subjected to external sinusoidal excitation forces with (a) $f=40 \mathrm{~Hz}, R=0.2 \mathrm{~mm}$ and (b) $f=20 \mathrm{~Hz}, R=0.1 \mathrm{~mm}$ 
The percentage reduction in the vibration amplitude is about $89.4 \%$ and almost independent of the desired position. On the other hand, as the frequency decreases the performance of vibration attenuation degrades but once again is independent of the desired position (see Fig. 8b). The percentage of vibration amplitude reduction decreases to $52.8 \%$ when the frequency decreases from 40 to $20 \mathrm{~Hz}$. It appears that the settling time is almost identical for the two sine cases and independent of the frequency value. Similarly, the beam tip is deflected by a specific amount and simultaneously subjected to an external random excitation force when the controller once again is employed to examine how fast it can reach the desired position and how well it can suppress the vibration. Fig. 9 shows the random excitation force as a function of time with maximum force value of $1(\mathrm{~N})$. Fig. 10 shows the comparison between the uncontrolled and controlled transient response under the random vibration when the controller is employed.

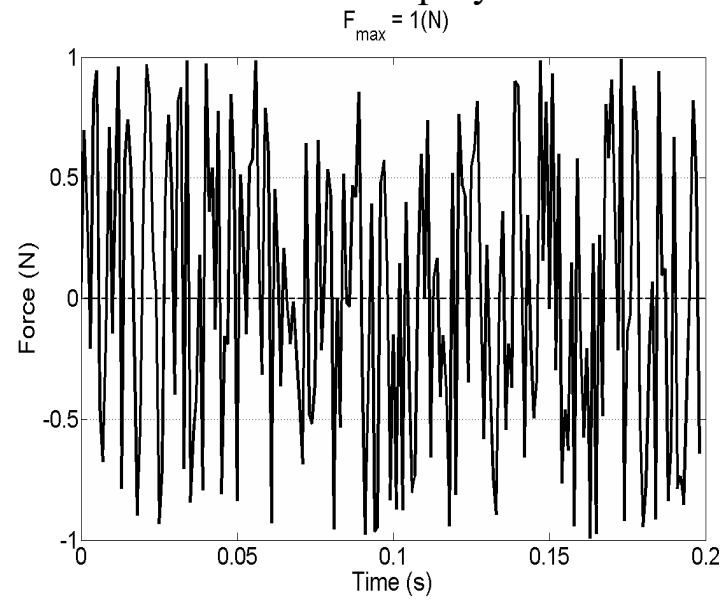

Figure 9: Time history of a random force

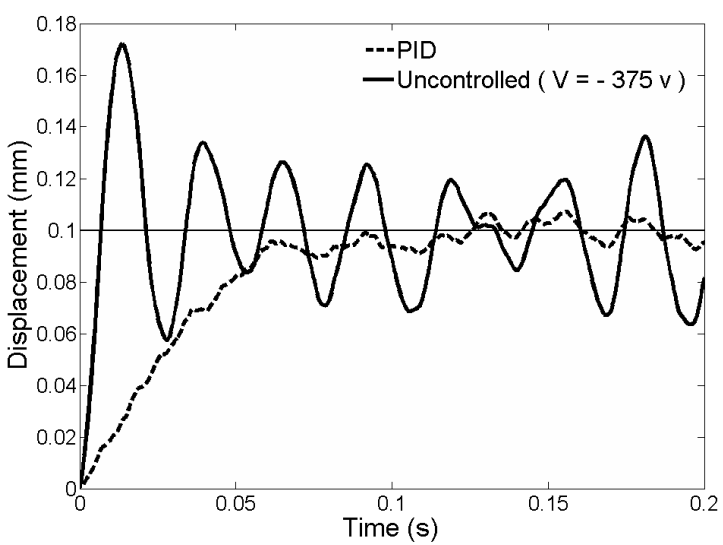

Figure 10: Effect of the controller on the vibration of the beam due to random force excitation

The desired position is $0.1 \mathrm{~mm}$. Once again, the results indicate that as the desired position increases or the beam tip deflects more, the control response curve becomes less distorted and reduces the settling time by a very small amount (see Fig. 10). It appears that regardless of the types of the external loads, the controller is able to reach the desired position almost at the identical settling time of about 0.12 seconds.

Fig. 11 shows the comparison between the uncontrolled and controlled transient responses when the beam tip is subjected to the external sine load at $40 \mathrm{~Hz}$ and zero desired position. The controller can perform only vibration suppression where the vibration reduction percentage is about $90.2 \%$. 
The actuator voltage values are stored for each time step during the transient analysis as the controller is employed and the beam tip is subjected to external sine loads at different frequencies and different desired positions (see Fig. 12).

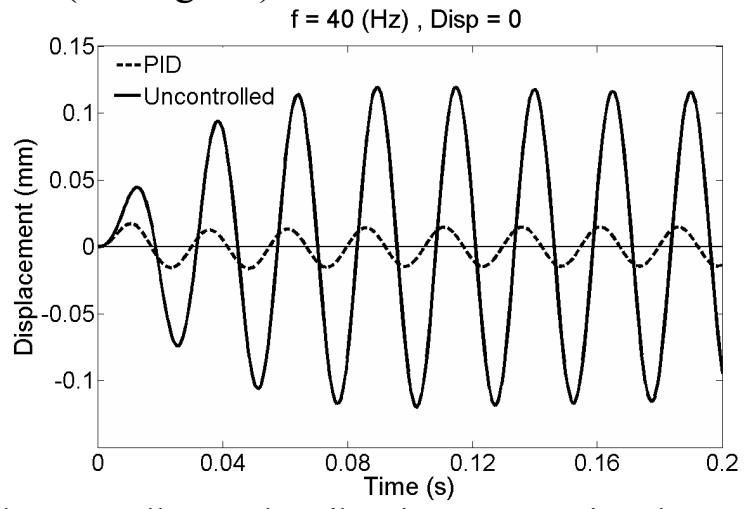

Figure 11: Effect of the controller on the vibration suppression due to forced sinusoidal excitation of the beam.

The actuator voltage values have two parts, one is DC part for positioning and the other is AC part for vibration suppression. The DC part of the control voltage changes linearly with desired position from 0.1 to $0.2 \mathrm{~mm}$; however, the AC part remains the same since the sinusoidal disturbance does not change (see Fig. 12a). It appears that the AC part of the actuator voltages have the same frequency as the applied external sinusoidal load. Fig. 12b shows the comparison between the required actuator voltage values for the external sine load having different frequencies while the desired position is held constant at $0.1 \mathrm{~mm}$. It is clear that the $\mathrm{AC}$ part of the actuator voltage curves represent the same frequencies as the applied external sine load; namely, $20 \mathrm{~Hz}$ and $40 \mathrm{~Hz}$, and the DC parts are the same since the desired position is the same (see Fig. 12b).

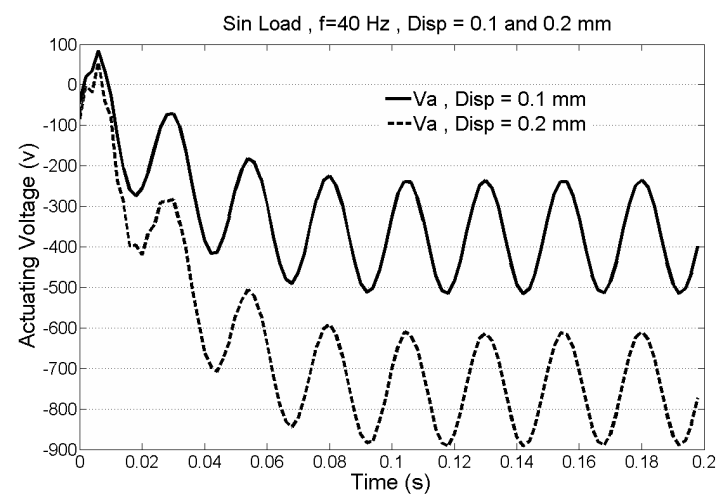

(a)

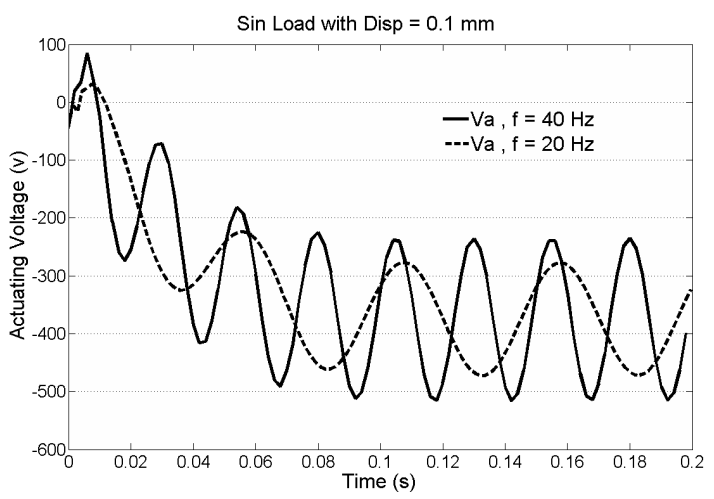

(b)

Figure 12: Actuator voltages (a) for different desired position (b) for different frequencies and identical desired position.

\section{Discussion on the results}

Characteristics and the best gain values of the controller are summarized in Table 2. The controller is used to examine how fast it can reach the desired position and how well it could be for vibration suppression. Results indicate that the steady state error reaches zero at about 0.12 seconds. On the other hand, when the controller is used to perform only vibration suppression, the vibration amplitude reduction percentage is $90.2 \%$ (see Table 2 ). 
Table 2: Characteristics and gain values for the controller

\begin{tabular}{ccc}
\hline Status & \% of V.S. & \% of steady state error \\
\hline Sine Load, $f=40 \mathrm{~Hz},(R=0.1 \mathrm{~mm})$ & $89.47 \%$ & $0 \%$ \\
Sine Load, $f=40 \mathrm{~Hz},(R=0.2 \mathrm{~mm})$ & $86.6 \%$ & $0 \%$ \\
Sine Load, $f=20 \mathrm{~Hz},(R=0.1 \mathrm{~mm})$ & $52.8 \%$ & $0 \%$ \\
Free Vibration, $(R=0.1 \mathrm{~mm})$ & $50.3 \%$ & $0 \%$ (at 0.1 second) \\
Sine Load, $f=40 \mathrm{~Hz},(R=0 \mathrm{~mm})$ & $90.2 \%$ & $0 \%$ \\
\hline
\end{tabular}

\section{Conclusions}

A FE environment with modelling, control design, and transient simulation capabilities is successfully developed and employed to study the performance of PID controller for FGPM beam with simultaneous vibration suppression and precision positioning. The controller satisfies both requirements of reaching the desired position in a very short time as well as performing vibration suppression.

Vibration suppression worsens for the controller as the frequency value decreases for the structure in this study. Vibration suppression gets better as the desired position increases. The controller is able to reach the desired position in about 0.12 seconds.

\section{References}

[1] Yu, T. and Zhong, Z., Bending analysis of a functionally graded piezoelectric cantilever beam. Science in China Series G: Physics, Mechanics and Astronomy, 50, 97-108, 2007.

[2] Şimşek, M., Static analysis of a functionally graded beam under a uniformly distributed load by Ritz method. International Journal of Engineering \& Applied Sciences, 1, 1-11, 2009.

[3] Wu, C., Kahn, M. and Moy, W., Piezoelectric ceramics with functional gradients: a new application in material design. Journal of the American Ceramic Society, 79, 809-812, 1996.

[4] Komijani, M., Kiani, Y., Esfahani, S.E. and Eslami, M.R., Vibration of thermo-electrically postbuckled rectangular functionally graded piezoelectric beams. Composite Structures, 98, 143-152, 2013.

[5] Amini, Y., Fatehi, P., Heshmati, M. and Parandvar, H., Time domain and frequency domain analysis of functionally graded piezoelectric harvesters subjected to random vibration: Finite element modeling. Composite Structures, 136, 384-393, 2016.

[6] Abdeljaber, O., Avci, O. and Inman, D.J., Active vibration control of flexible cantilever plates using piezoelectric materials and artificial neural networks. Journal of Sound and Vibration, 363, 3353, 2016.

[7] Ma, K. and Melcher, J., Adaptive control of structural acoustics using intelligent structures with embedded piezoelectric patches. Journal of Vibration and Control, 9, 1285-1302, 2003.

[8] Chhabra, D., Bhushan, G. and Chandna, P., Optimal placement of piezoelectric actuators on plate structures for active vibration control via modified control matrix and singular value decomposition 
approach using modified heuristic genetic algorithm. Mechanics of Advanced Materials and Structures, 23, 272-280, 2016.

[9] Karagülle, H., Malgaca, L. and Öktem, H.F., Analysis of active vibration control in smart structures by ANSYS. Smart Materials and Structures, 13, 661-667, 2004.

[10] Irschik, H., A review on static and dynamic shape control of structures by piezoelectric actuation. Engineering Structures, 24, 5-11, 2002.

[11] Russ, R., Ma, K. and Ghasemi-Nejhad, M.N., A finite element analysis approach with integrated PID control for simultaneous precision positioning and vibration suppression of smart structures. In Smart Structures and Materials (616617-616617), International Society for Optics and Photonics, 2006.

[12] Phung-Van, P., Nguyen-Thoi, T., Le-Dinh, T. and Nguyen-Xuan, H., Static and free vibration analyses and dynamic control of composite plates integrated with piezoelectric sensors and actuators by the cell-based smoothed discrete shear gap method (CS-FEM-DSG3). Smart Materials and Structures, 22, 095026 (17pp), 2013.

[13] Wang, C.M., Ang, K.K. and Ajit, A., Shape control of laminated cantilevered beams with piezoelectric actuators. Journal of intelligent material systems and structures, 10, 164-175, 1999.

[14] ABAQUS Documentation and User Manual, Version 6.12., Simulia, Dassault Systèmes, 2012. 